\title{
Polymorphism, coupling interval and prematurity index in dogs with degenerative mitral valve disease and ventricular arrhythmias
}

\author{
Elizabeth Regina Carvalho ${ }^{1}$. Roberto Andrés Navarrete Ampuero ${ }^{1} \cdot$ Giovana Laís Ruviaro Tuleski ${ }^{2}$. \\ Aparecido Antonio Camacho' ${ }^{1}$ Marlos Gonçalves Sousa ${ }^{2}$
}

Received: 7 December 2017 / Accepted: 9 March 2018 / Published online: 13 March 2018

๑) Springer Science+Business Media B.V., part of Springer Nature 2018

\begin{abstract}
Ventricular arrhythmias (VA) are a recognized concern in dogs with degenerative mitral valve disease (DMVD). The coupling interval (CI) and the prematurity index (PI) have been shown to accurately differentiate between benign and malignant VA in people, where ventricular arrhythmias are known to be associated with an increased risk of development of signs of heart failure or sudden death. In this study, we characterized ventricular arrhythmias in dogs with symptomatic and asymptomatic DMVD. Seventy dogs with naturally-occurring DMVD and ventricular arrhythmias were retrospectively studied. A crosssectional investigation including dogs with either symptomatic (stages $C / D ; n=41$ ) or asymptomatic (stages $B 1 / B 2 ; n=29$ ) DMVD was performed. Electrocardiographic tracings were reviewed to calculate both the CI and PI. In eight dogs these indices were compared with those obtained from both a Holter recording and a standard ECG tracing and no statistical differences were found (CI, $p=0.97$; PI, $p=0.17)$. Even though CI and PI were determined in all animals enrolled in the study, VPC characteristics were only compared between symptomatic and asymptomatic dogs when a 24-h Holter recording was available $(\mathrm{n}=49)$. The PI was different $(p=0.01)$ between symptomatic $(0.65 \pm 0.17)$ and asymptomatic $(0.56 \pm 0.18)$ dogs, but CI was considered similar $(p=0.91)$. Also, the symptomatic dogs had more polymorphic VPC $(p=0.002)$ and supraventricular arrhythmias $(p=0.0002)$ than the asymptomatic animals. Polymorphism, and repeating patterns of ventricular premature complexes, were characteristics frequently present in overtly symptomatic animals affected by mitral endocardiosis.
\end{abstract}

Keywords Electrocardiography $\cdot$ Endocardiosis $\cdot$ Ventricular premature complexes $\cdot$ Polymorphic $\cdot$ Supraventricular arrhythmia

\section{Abbreviations}

AF Atrial fibrillation

CI Coupling interval

DMVD Degenerative mitral valve disease

VPC Ventricular premature complex

PI Prematurity index

PVT Paroxysmal ventricular tachycardia

SCL Sinus cycle length

SVA Supraventricular arrhythmias

Elizabeth Regina Carvalho

beth_rcarvalho@hotmail.com

1 College of Veterinary Medicine and Agricultural Sciences, São Paulo State University (UNESP) Jaboticabal, Via de Acesso Professor Paulo Donato Castellane, s/n, 14884-900 Jaboticabal, São Paulo, Brazil

2 Department of Veterinary Medicine, Federal University of Paraná (UFPR), Rua dos Funcionários, 1540, 80035-050 Curitiba, Paraná, Brazil
SVT Supraventricular tachycardia

TI Tricuspid insufficiency

VA Ventricular arrhythmias

VT Ventricular tachycardia

\section{Introduction}

Ventricular arrhythmias (VA) have been shown to be a concern in dogs with degenerative mitral valve disease (DMVD), even in initial stages of disease (Crosara et al. 2010), and sudden cardiac death has been described in DMVD dogs without clinical signs (Borgarelli et al. 2008). Normal ventricular function depends on synchronous activation of the ventricular myocardium in order to maximize the systolic pump function. Electrical activation originating within ventricular myocardium resulting in ventricular premature complexes (VPC) reduces diastolic time, which 
reduces ventricular filling, cardiac output and coronary perfusion.

In human patients with mitral valve prolapse, a disease closely related to DMVD in dogs, it is assumed that VA play an important role in the development of clinical signs and sudden cardiac death (Basso et al. 2015). For this reason, the coupling interval (CI), defined as the R-R interval between the VPC and the preceding sinus complex, and the prematurity index (PI), defined as the ratio of the $\mathrm{CI}$ to the preceding R-R interval of the sinus cycle just before the VPC, have been studied as "novel" techniques for distinguishing between benign and malignant VA. Previous reports have suggested that short CI results in polymorphic malignant ventricular tachycardia or ventricular fibrillation (Haissaguerre et al. 2002; Viskin et al. 2005; Knecht et al. 2011). However, Igarashi et al. (Igarashi et al. 2012) found the PI to be the only independent determinant of malignant VA.

In this study, the authors characterized ventricular arrhythmias in dogs with symptomatic or asymptomatic DMVD. Therefore, the aims of this research were twofold: (1) to investigate how both CI and PI perform in dogs with degenerative mitral valve disease and VA; and (2) to evaluate the characteristics of VPCs, including site of origin, frequency and morphology, and how they affect CI and PI.

\section{Animals, materials and methods}

This was a retrospective, cross-sectional study design, and was conducted at two Veterinary Teaching facilities. The study was approved by the institutional Animal Care and Use Committee (protocol 12,476/16).

\section{Animals}

Medical records of dogs presented for consultation to the Cardiology Sections between January 2012 and October 2016, either because of the presence of a heart murmur, or because of clinical signs indicating a cardiovascular disorder (e.g. cough, dyspnea, exercise intolerance), were reviewed.

Inclusion criteria: dogs with naturally-occurring degenerative mitral valve disease confirmed by echocardiographic examination; patients were filtered for the presence of premature ventricular complexes documented either by $24-\mathrm{h}$ Holter monitoring or a standard electrocardiographic (ECG) tracing.

Exclusion criteria: dogs were excluded if they had suspected or confirmed neoplasia, had received antiarrhythmic drugs (classes 1 to 4), had an unconventional ECG recording (e.g. ECG recorded in a position other than the right lateral recumbency), or if they had congenital heart diseases, or acquired cardiovascular disorders that primarily or secondarily affect the mitral valve (e.g. bacterial endocarditis, dilated cardiomyopathy).

Based on clinical history, the animals were divided in two groups: asymptomatic (AG), including DMVD dogs in stages B1 and B2; or symptomatic (SG), including dogs in stages C and D (Atkins et al. 2009).

\section{Electrocardiographic analyses}

A three-channel 24-h Holter ${ }^{1}$, with four electrodes placed at the level of the base and apex in each hemithorax, was conducted while the dogs were kept at home. One operator, blinded to the patients, manually edited all data analyses, checking the QRS templates, including possible misinterpretation for horizontal and vertical resolution, checking the digital 24-h editing program. Measurements, as well as analyses, were made on channel 1 (positive pole toward the apex of the left ventricle).

A high-quality 10 -lead $\mathrm{ECG}^{2}$ recording, with the electrodes placed as described elsewhere and animal positioned on right lateral recumbency, was recorded in the hospital with a minimal recording time of $3 \mathrm{~min}$. The measurements were made on lead II (inferior frontal plane lead, positive pole toward the apex of the left ventricle).

Using either three-channel 24-h Holter or 10-lead standard ECG recordings, the coupling interval (CI) and the sinus cycle length (SCL), defined as the R-R interval of the sinus cycle just before the VPC, were determined as described elsewhere (Igarashi et al. 2012; Kim et al. 2014). The prematurity index (PI) was calculated as the ratio of CI to SCL (Fig. 1).

Five VPCs per dog were chosen at random, all measurements were made five times, the highest and lowest values were excluded and a mean of three measurements was used for statistical analysis. When the randomization included a run of paroxysmal ventricular tachycardia (PVT), the first VPC of the run and the SCL from the sinus beats before the episode were used. Dogs with less than 5 VPCs recorded had all ventricular premature beats measured, and a mean was obtained. All analyses were made by the same evaluator, who was blinded to the group distribution.

The clinical history, DMVD stage, echocardiographic data, and the device used (either Holter or standard ECG) were also recorded.

Arrhythmia characteristics were only evaluated when a 24-h Holter recording was available, and were divided into two main groups: ventricular arrhythmias (VPC, couplets, triplets, ventricular bigeminy, trigeminy, quadrigeminy, pentageminy or hexageminy, and sustained or paroxysmal

\footnotetext{
${ }^{1}$ CardioLight, Cardios Sistema, São Paulo, Brazil.

${ }^{2}$ ECGPC TEB, Tecnologia Eletrônica Brasileira, São Paulo, Brazil.
} 


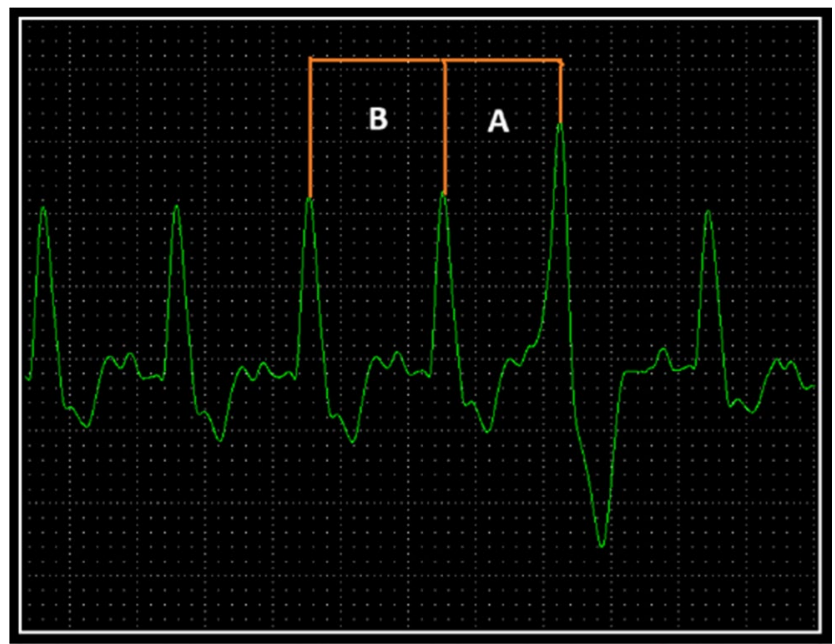

Fig. 1 Schematic representation indicating the measurement of the coupling interval (a) and the sinus cycle length (b), as well as the calculation of the prematurity index (A:B) in lead II ECG tracing

ventricular tachycardia (VT), and supraventricular arrhythmias (sustained or paroxysmal supraventricular tachycardia (SVT), atrial fibrillation (AF). VT was defined as a sequence of more than three consecutive large QRS complexes, with HR $>180$ beats/min and atrioventricular dissociation. SVT was defined as a sequence of more than three consecutive narrow QRS complexes with regular RR intervals, HR $>200$ beats/min and no evidence of $\mathrm{P}$ wave. Any arrhythmia with a duration less than $30 \mathrm{~s}$ was defined as paroxysmal. Holter recordings are notoriously poor for correctly identifying true supraventricular arrhythmias, for this reason, the presence of a single atrial premature complex (APC) was not included in analyses.

Additionally, the following criteria of VA were documented using only the 24-h Holter monitoring: (1) site of origin of VPC: left ventricle (first deflection negative), right ventricle (first deflection positive), or both. (2) VPC morphology: monomorphic (unifocal - same QRS morphology and polarity throughout the exam) or polymorphic (multifocal - QRS morphology changing with regard to height and/ or polarity). (3) VPC frequency: isolated VPC, repeating patterns (couplets, triplets, bigeminy, trigeminy, quadrigeminy, pentageminy or hexageminy) or VT.

\section{Echocardiographic analyses}

The echocardiographic exams ${ }^{3}$ of all animals included on study were also reviewed in order to retrospectively assess the following measurements: left atrium-to-aorta ratio (LA:Ao), left ventricular internal dimension at end-diastole-to-aorta ratio (LVIDd:Ao), left ventricular internal dimension at end-systole-to-aorta ratio (LVIDs:Ao), fractional shortening (FS), ratio of early-to-late left ventricular filling velocities (E:A), and isovolumic relaxation time (IVRT). Left atrium and aorta diameters were measured at right parasternal window using short axis two-dimensional images at the very last frame before aortic valve opening, and left ventricular internal diameters in systole and diastole were assessed by M-mode, also in short axis images (Chetboul and Tissier 2012). The assessment of E:A and IVRT were accomplished at left parasternal window using apical four and five chambers views, respectively (Chetboul and Tissier 2012). The FS was calculated by the formula: $\mathrm{FS}=[(\mathrm{LVIDd}-\mathrm{LVIDs}) / \mathrm{LVIDd}] * 100$, as described elsewhere.

\section{Statistical analyses}

Statistical processing was performed using commercially available statistical software ${ }^{4}$. The Shapiro-Wilk test was used to investigate the normal distribution of data. Comparisons between groups were accomplished by either Mann-Whitney test or Student's $t$-test according to distribution. The contingency was evaluated using either Fisher's exact test or the Chi square when three or more categories were compared. The correlation between echocardiographic and electrocardiographic parameters was assessed by the Spearman test, or Pearson test, for parametric data.

When the CI and PI were evaluated according to VPC categories resulting in three different groups, data was submitted again to the Shapiro-Wilk normality test. Posteriorly, parametric data was compared using a one-way analysis of variance (ANOVA) followed by the Tukey test, while for non-parametric data the comparison was accomplished with the Kruskal Wallis test, followed by the Dunn post-test. Statistical significance was defined as $p<0.05$.

\section{Results}

\section{Animals}

A total of $70 \mathrm{dogs}$ met the inclusion/exclusion requirements and were recruited for this study. Twenty-nine were asymptomatic (AG) and forty-one had clinical signs ascribed to cardiac disease (SG). The population enrolled in AG included DMVD dogs in stages B1 (72.4\%) and B2 (27.6\%), whereas SG was composed of DMVD dogs in stages C (92.7\%) and $\mathrm{D}(7.3 \%)$. No differences existed between groups with regard to sex, body weight, or age. The characteristics of the studied population are summarized in Table 1.

\footnotetext{
${ }^{4}$ Prism Windows 5.0, GraphPad Software, CA, USA.
} 
Table 1 Characteristics of the studied population subdivided according to the presence or absence of clinical signs attributable to heart failure. The sample size is shown in parentheses

\begin{tabular}{lllc}
\hline & AG $(29)$ & SG (41) & $P$ \\
\hline Age, years (63) & $7-15($ median 12y) & $7-18$ (median 12y) & $0.20^{\ddagger}$ \\
Weight, Kg (68) & $3.2-19.2$ & $2.7-26$ & $0.87^{*}$ \\
Sex (70) & Males: $12(41.4 \%)$ Females: $17(58.6 \%)$ & Males: $25(61 \%)$ Females: $16(39 \%)$ & $0.14^{\ddagger \ddagger}$ \\
DMVD Stage (70) & B1: $21(72.4 \%)$ B2: $8(27.6 \%)$ & C: $38(92.7 \%)$ D: $3(7.3 \%)$ & Mean \pm SD \\
LA:Ao (70) & Mean \pm SD & $2.38 \pm 0.70$ & $<.48 \pm 0.61$ \\
LVIDd:Ao (69) & $1.38 \pm 0.22$ & $1.32 \pm 0.36$ & $0.001^{\ddagger}$ \\
LVIDs:Ao (69) & $1.77 \pm 0.34$ & $46 \pm 8$ & $0.01^{\ddagger}$ \\
FS, \% (69) & $1.06 \pm 0.22$ & $1.65 \pm 1.04$ & $0.22^{*}$ \\
E:A (60) & $40 \pm 8$ & $43 \pm 22$ & $0.06^{\ddagger}$ \\
IVRT, ms (31) & $1.30 \pm 0.45$ & & \\
\hline
\end{tabular}

* Student’s $t$-test. ${ }^{\ddagger}$ Mann-Whitney test. ${ }^{\ddagger \ddagger}$ Fisher’s exact test

$A G$ Asymptomatic group, $S G$ Symptomatic group, $S D$ Standard deviation, LA:Ao, Left atrium-to-aorta ratio, LVIDd:Ao, Left ventricular internal dimension at end-diastole-to-aorta ratio, LVIDs:Ao Left ventricular internal dimension at end-systole-to-aorta ratio, FS Fractional shortening, $E: A$ ratio of early-to-late left ventricular filling velocities, IVRT isovolumetric relaxation time

\section{Comparison between Holter and ECG analyses}

In order to investigate whether measurements obtained from 3-channel 24-h Holter recordings and computer-based 10-lead ECG recordings could be analyzed together, we compared the results obtained in eight dogs from SG of this study, in which records of VPC on either 24-h Holter and standard ECG were available. Measurements of CI, SCL and PI were obtained from the Holter's channel 1 and ECG lead II tracings. Data was normally-distributed and no differences existed between the two devices for $\mathrm{CI}(p=0.97)$, PI $(p=0.17)$ and SCL $(p=0.11)$.

\section{Coupling interval, sinus cycle length and prematurity index}

The overall results include measurements obtained from Holter recordings $(n=49)$ and standard ECG tracings $(\mathrm{n}=21)$. AG data was obtained from Holter recordings in 23 dogs and ECG tracings in 6 animals, while SG results were obtained from Holter and ECG recordings in 26 and 15 dogs, respectively.

The PI was different $(p=0.01)$ between SG $(0.65 \pm 0.17)$ and AG $(0.56 \pm 0.18)$ dogs, but CI was considered similar $(p=0.91)$. The SCL was different $(p=0.0085)$ among AG $(513 \pm 129 \mathrm{~ms})$ and SG $(433 \pm 116 \mathrm{~ms})$. Also, the mean heart rate analyzed only in dogs with 24-h Holter monitoring was significantly different $(p=0.03)$ between AG $(107 \pm 27 \mathrm{bpm})$ and SG $(124 \pm 24 \mathrm{bpm})$. The mean heart rate did not correlate with $\mathrm{CI}(\mathrm{R}=-0.09,95 \%$ confidence interval $=-0.37$ to 0.19 ), however, the mean heart rate correlated positively with PI $(R=0.34,95 \%$ confidence interval $=0.06$ to 0.58$)$. Results are summarized in Table 2.

There was no correlation between CI and echocardiographic parameters. Correlation coefficients obtained
Table 2 Coupling interval, sinus cycle length and prematurity index obtained from dogs with degenerative mitral valve disease and ventricular arrhythmias

\begin{tabular}{lllllll}
\hline & CI $(\mathrm{ms})$ & \multicolumn{3}{c}{ SCL $(\mathrm{ms})$} & & PI \\
\hline & AG & SG & AG & SG & AG & SG \\
Min-Max & $170-430$ & $180-495$ & $270-830$ & $200-820$ & $0.32-1.0$ & $0.36-1.2$ \\
IQR & $231.5-315.0$ & $248.5-291.5$ & $425.0-608.5$ & $356.5-513.5$ & $0.43-0.65$ & $0.54-0.80$ \\
Median & 263 & 263 & 466 & 411 & 0.51 & 0.61 \\
Mean \pm SD & $275.5 \pm 65.2$ & $271.1 \pm 58.6$ & $513.6 \pm 129.5$ & $433.6 \pm 116$ & $0.56 \pm 0.18$ & $0.65 \pm 0.17$ \\
SE & 12.2 & 9.15 & 24.05 & 18.11 & 0.03 & 0.02 \\
$95 \%$ CI & $250.5-300.6$ & $252.6-289.6$ & $464.4-562.9$ & $397.0-470.2$ & $0.49-0.63$ & $0.60-0.71$ \\
$P$ & $0.9145^{*}$ & & $0.0085^{\ddagger}$ & & $0.015^{*}$ & \\
\hline
\end{tabular}

* Mann-Whitney test. ${ }^{*}$ Student's $t$-test

$A G$ Asymptomatic group, SG Symptomatic group, $C I$ Coupling interval, SCL Sinus cardiac length, PI Prematurity index, $Q T$ QT interval, $Q T I$, QT index, Min-Max, Minimum and maximum values, $I Q R$, interquartile range, $S D$, Standard deviation, $S E$, Standard error, $95 \%$ CI, 95\% confidence interval 
between CI, SCL, PI and echocardiographic data are shown in Table 3. The SCL is negatively correlated with LA:Ao, while PI is positively correlated with echocardiographic parameters of volume overload, such as LA:Ao and LVIDs:Ao.

\section{Arrhythmia characteristics}

Only Holter recordings were used to assess arrhythmia characteristics $(n=49)$. The number of VPCs recorded during the 24-h Holter were different $(p=0.01)$ between AG (1 to 132 beats) and SG (1 to 6011 beats). When dogs were separated according to LA:Ao (greater than 1.6 or equal/smaller than 1.6), the occurrence of SVA was significantly associated with enlarged left atrium $(p=0.04)$. The incidence of supraventricular arrhythmias was also considered different between AG and SG $(p=0.0002)$ (Table 4). No animals exhibited sustained supraventricular

Table 3 Correlation coefficients obtained between coupling interval, sinus cardiac length or prematurity index and the echocardiographic data in dogs with naturally-occurring degenerative mitral valve disease and ventricular arrhythmias

\begin{tabular}{lll}
\hline & $\mathrm{R}$ & $95 \%$ CI \\
\hline CI $v s$ & & \\
LA:Ao & $0.03^{*}$ & -0.21 to 0.27 \\
LVIDd:Ao & $-0.19^{*}$ & -0.42 to 0.05 \\
LVIDs:Ao & $-0.06^{*}$ & -0.31 to 0.18 \\
FS & $-0.15^{*}$ & -0.38 to 0.09 \\
E:A & $-0.05^{*}$ & -0.30 to 0.21 \\
IVRT & $0.20^{*}$ & -0.17 to 0.52 \\
SCL $v s$ & & \\
LA:Ao & $-0.34^{\ddagger}$ & -0.55 to -0.10 \\
LVIDd:Ao & $-0.20^{*}$ & -0.42 to 0.05 \\
LVIDs:Ao & $-0.10^{\ddagger}$ & -0.33 to 0.14 \\
FS & $-0.12^{\ddagger}$ & -0.35 to 0.11 \\
E:A & $0.02^{*}$ & -0.23 to 0.28 \\
IVRT & $-0.21^{\ddagger}$ & -0.52 to 0.15 \\
PI $v s$ & & \\
LA:Ao & & 0.11 to 0.54 \\
LVIDd:Ao & $0.35^{*}$ & -0.03 to 0.44 \\
LVIDs:Ao & $0.21^{*}$ & 0.02 to 0.48 \\
FS & $0.27^{*}$ & -0.14 to 0.33 \\
E:A & $0.10^{*}$ & -0.21 to 0.30 \\
IVRT & $0.05^{*}$ & -0.001 to 0.64 \\
\hline & $0.36^{*}$ &
\end{tabular}

* Spearman test. ${ }^{\ddagger}$ Pearson test

$R$ correlation coefficient, 95\% CI, 95\% confidence interval, $C I$ Coupling interval, SCL Sinus cardiac length, PI Prematurity index, vs Versus, LA:Ao, Left atrium-to-aorta ratio, LVIDd:Ao, Left ventricular internal dimension at end-diastole-to-aorta ratio, LVIDs:Ao, Left ventricular internal dimension at end-systole-to-aorta ratio, $F S$ Fractional shortening, $E: A$, ratio of early-to-late left ventricular filling velocities, IVRT Isovolumetric relaxation time tachycardia, and two dogs showed atrial fibrillation, both from $\mathrm{SG}$ (one at stage $\mathrm{C}$ and another at stage $\mathrm{D}$ ).

Moreover, the morphology of VPCs was different among groups $(p=0.002)$. The symptomatic group had more polymorphic ventricular arrhythmias, and episodes of repeating patterns VPC or PVT than asymptomatic dogs.

With respect to the origin of a VPCs, there were no significant differences between symptomatic and asymptomatic dogs $(p=0.22)$. In AG, seven dogs $(30.4 \%)$ had concurrent tricuspid insufficiency (TI), of these, four (57.1\%) had VPCs arising from the right ventricle or both ventricles, while five dogs without echocardiographic signs of TI had VPCs arising from the right ventricle. In SG, twenty patients (76.9\%) also had TI, of these, eight (40\%) had VPCs arising from the right ventricle or both ventricles.

The coupling interval and prematurity index seems to be directly influenced by the occurrence of supraventricular arrhythmias. The CI was considered shorter $(p=0.05)$ in patients that had SVA $(254 \pm 44 \mathrm{~ms})$ as compared to those without SVA ( $284 \pm 67 \mathrm{~ms})$. The CI did not differ according to the characteristics of the VPCs. However, VPCs with greater PI, were documented more frequently during paroxysmal ventricular tachycardia or repeating pattern episodes as compared to only isolated premature beats. Detailed results are shown in Table 4 and Fig. 2 shows the

Table 4 Descriptive analyses of ventricular arrhythmias documented by 24-h Holter recordings in dogs with naturally-occurring degenerative mitral valve disease. Values in parentheses represent the sample size

\begin{tabular}{|c|c|c|c|}
\hline & AG (23) & SG (26) & $P$ \\
\hline \multicolumn{4}{|c|}{ Supraventricular arrhythmias } \\
\hline Yes & $8.70 \%(2)$ & $57.70 \%(15)$ & $0.0002^{*}$ \\
\hline No & $91.30 \%(21)$ & $42.30 \%(11)$ & \\
\hline \multicolumn{4}{|l|}{ PVCs site of origin } \\
\hline Left ventricle & $60.87 \%(14)$ & $69.23 \%(18)$ & $0.22^{\ddagger}$ \\
\hline Right ventricle & $26.08 \%(6)$ & $7.70 \%(2)$ & \\
\hline Both ventricles & $13.05(3)$ & $23.07(6)$ & \\
\hline \multicolumn{4}{|l|}{ PVCs morphology } \\
\hline Monomorphic & $91.30 \%(21)$ & $53.84 \%(14)$ & $0.002 *$ \\
\hline Polymorphic & $8.70 \%(2)$ & $46.16 \%(12)$ & \\
\hline \multicolumn{4}{|l|}{ PVCs frequency } \\
\hline Isolated & $86.95 \%(20)$ & $53.84 \%(14)$ & $0.04^{\ddagger}$ \\
\hline Repeating patterns & $8.70 \%(2)$ & $34,61 \%(9)$ & \\
\hline PVT & $4.35 \%(1)$ & $11.55 \%(3)$ & \\
\hline
\end{tabular}

* Fisher's exact test. ${ }^{\ddagger}$ Chi square test

$A G$ Asymptomatic group, $S G$ Symptomatic group, PVCs, Premature ventricular complexes, Repeating patterns, include premature ventricular complexes in couplets, triplets, bigeminy, trigeminy, quadrigeminy, pentageminy or hexageminy, $P V T$, Paroxysmal ventricular tachycardia 


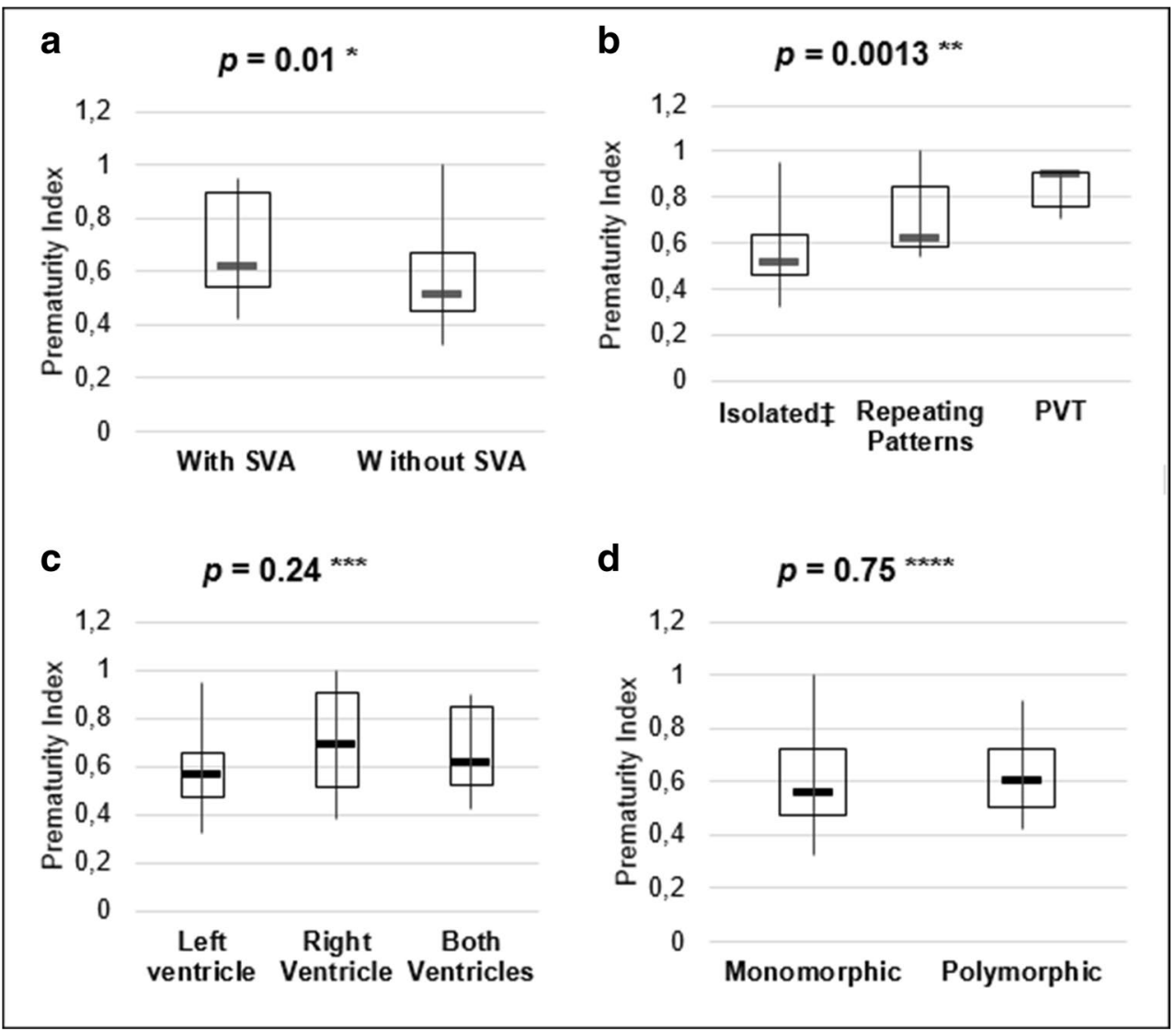

Fig. 2 Box plots corresponding to the analyses of the prematurity index in relation to the characteristics of either premature ventricular beats or supraventricular arrhythmia. (a) PI was significantly lower when supraventricular arrhythmias were not present; (b) PI was significantly greater in dogs with repeating patterns and paroxysmal ventricular tachycardia as compared to animals in which only isolated premature ventricular contractions were documented; (c) The origin of VPCs did not affect the PI; (d) Patients with either monomorphic

comparison of PI with respect to the characteristics of VPCs or the occurrence of supraventricular arrhythmia.

\section{Discussion}

Studies evaluating the coupling interval in veterinary patients are lacking. Although many investigations do exist in people with ventricular tachycardia, results are conflicting when it comes to the usefulness of the $\mathrm{CI}$ to distinguish between benign and malignant forms of ventricular arrhythmia. While some studies suggest that $\mathrm{CI}$ is similar between those types of arrhythmia (Del Carpio Munoz et al. 2011; Igarashi et al. 2012; Kurosaki et al. 2013), other, documented a relationship between shorter CI, malignant ventricular tachycardia and sudden cardiac death (Sun et al. 2003; Viskin et al. 2005; Kim et al. 2014). The coupling interval of VA in asymptomatic and symptomatic DMVD dogs was or polymorphic ventricular premature beats had similar PI. * MannWhitney test. ** Kruskal-Wallis test, followed by Dunn test; ${ }^{*}$ statistical difference between PI of isolated PVCs and repeating patterns, also between PI of isolated PVCs and PVT $(p<0.05)$; *** ANOVA, followed by Tukey test; **** Student's $t$-test. SVA, supraventricular arrhythmias (SVT or AF); Repeating Patterns, include episodes of couplets, triplets, bigeminy, trigeminy, quadrigeminy, pentageminy or hexageminy; PVT, paroxysmal ventricular tachycardia

similar in our investigation, and did not differ between polymorphic or monomorphic VPC.

The progression of heart disease leads to a decrease in parasympathetic tone and an increase in sympathetic tone, as a compensatory neuroendocrine mechanism. Therefore, the heart rate variability decreases in individuals with heart failure (Piccirillo et al. 2009; Oliveira et al. 2012). As expected, our study demonstrates that symptomatic dogs have significantly higher heart rates. A negative correlation between an echocardiographic parameter of cardiac remodeling (LA:Ao) and sinus cycle length was shown. In our study, the SCL was significantly shorter - ie heart rate was faster - in overtly symptomatic animals, leading to a greater PI in that group as compared to the asymptomatic dogs, whilst CI was comparable.

Igarashi and others (Igarashi et al. 2012) showed that in human beings, the PI was the only independent determinant of polymorphic QRS and ventricular fibrillation, 
in spite of the CI being similar to the control group. However, in their study, patients with polymorphic ventricular tachycardia were those with the lowest prematurity index, while our findings suggesting that PI is not a determinant of the polymorphic VPC in dogs affected by DMVD.

The weak positive correlation coefficient obtained between PI and echocardiographic surrogates of cardiac remodeling (LA:Ao, $\mathrm{R}=0.35$; LVIDs:Ao, $\mathrm{R}=0,27$ ) might have been underestimated due to the unequal number of dogs in groups classified according to DMVD stage. The requirement of an ECG recording in right lateral recumbency probably explains why only a small number of dogs in stage D were enrolled in this study, since those patients are usually in respiratory distress and hemodynamically unstable, therefore recordings will be made in non-traditional positions to minimize stress.

Our findings show that symptomatic dogs had enlarged left atriums and more SVA in comparison with asymptomatic animals. Therefore, a relationship between atrial overload and an increased predisposition to arrhythmias might be anticipated. In a study that assessed arrhythmias in different stages of DMVD, Crosara et al. (2010) found that runs of SVA were significantly more frequent in dogs with an advanced form of DMVD.

Many theories have been proposed to explain triggering mechanisms of VA in mitral insufficiency, suggestions include chordal traction by redundant mitral leaflets (Barlow, M.B., Bosman 1965), endocardial fibrosis (Mason et al. 1978; Savage et al. 1983), myocardial hypoxemia (Shepard et al. 1985), or even ventricular dilation and stretch with increased myocardial wall strain (Hansen et al. 1990). Conversely, even dogs with no cardiac remodeling (stage B1) had VA. Interestingly, the right ventricle was also demonstrated to be the origin of VPCs even in dogs without tricuspid insufficiency (five animals from AG). These findings suggest that VA in dogs with DMVD might have a multifactorial etiology regardless of class of heart failure, as previously proposed by Crosara et al. (2010).

In symptomatic dogs, VPCs are more commonly associated with polymorphism and repeating patterns/PVT episodes than in asymptomatic subjects. Many studies have reported that multifocal and more frequent VPCs are closely associated with left ventricular dysfunction, arrhythmogenic cardiomyopathy, malignant forms of ventricular tachycardia, as well as a poor prognosis (Duffee et al. 1998; Shiraishi et al. 2002; Takemoto et al. 2005; Rhee et al. 2006; Kanei et al. 2008; Sheldon et al. 2010; Del Carpio Munoz et al. 2011; Igarashi et al. 2012; Lee et al. 2014). In veterinary patients it remains unclear if polymorphism and a higher frequency of VPCs could play a role in left ventricular dysfunction. Based on our results, polymorphism as well as repeating pattern VPCs, were more frequent VPC characteristics than prematurity in overtly symptomatic animals affected by mitral endocardiosis.

In addition, the coexistence of SVA resulted in premature ventricular beats with a lower coupling interval, although, the PI suggests that ventricular arrhythmias were less premature during SVA episodes. Similarly, when considering PI, repeating pattern VPCs/PVT resulted in less premature VPCs in DMVD dogs. The authors believe these findings could be explained by the heart rate influence on prematurity index, and caution should be take when interpreting isolated PI values of VA in dogs with DMVD, once heart rate is chronically elevated in such patients.

The results of this study should be interpreted in the context of its limitations. This was a retrospective investigation and accepts the inherent criticisms of the methodology. The unequal sample size with regard to DMVD stage, and the small number of stage D dogs, limits the stratification of $\mathrm{CI}$ and PI. The differences in heart rate due to sympathetic activation in symptomatic animals has a fundamental impact in the prematurity index. Furthermore, this study did not investigate whether polymorphism, repeating patterns or less premature VPCs are associated with a worse prognosis and a reduced survival in dogs affected by mitral endocardiosis.

\section{Conclusion}

Our major findings are as follows. (1) Although CI was comparable between the symptomatic and asymptomatic groups, VPCs were less premature in symptomatic dogs; (2) The symptomatic dogs had more supraventricular arrhythmias, polymorphic ventricular arrhythmias, and episodes of repeating patterns VPC or PVT than asymptomatic dogs; (3) The coupling interval and the prematurity index seem to be directly influenced by the occurrence of supraventricular arrhythmias. Also, greater PI (less premature VPCs) was documented more frequently during paroxysmal ventricular tachycardia or repeating pattern episodes as compared to only isolated premature beats.

\section{Compliance with ethical standards}

Conflict of interest The authors do not have any conflicts of interest to disclose.

\section{References}

Atkins C, Bonagura J, Ettinger S et al (2009) Guidelines for the diagnosis and treatment of canine chronic valvular heart disease. J Vet Intern Med 23:1142-1150. https://doi. org/10.1111/j.1939-1676.2009.0392.x

Barlow MB, Bosman MB (1965) Aneurysmal protrusion of the posterior leaflet of the mitral valve. Am Heart J 71:166-178 
Basso C, Perazzolo Marra M, Rizzo S et al (2015) Arrhythmic Mitral Valve Prolapse and Sudden Cardiac Death. Circulation 132:556566. https://doi.org/10.1161/CIRCULATIONAHA.115.016291

Borgarelli M, Savarino P, Crosara S et al (2008) Survival characteristics and prognostic variables of dogs with mitral regurgitation attributable to myxomatous valve disease. J Vet Intern Med 22:120-128. https://doi.org/10.1111/j.1939-1676.2007.0008.x

Chetboul V, Tissier R (2012) Echocardiographic assessment of canine degenerative mitral valve disease. J Vet Cardiol 14:127-148. https ://doi.org/10.1016/j.jvc.2011.11.005

Crosara S, Borgarelli M, Perego M et al (2010) Holter monitoring in 36 dogs with myxomatous mitral valve disease. Aust Vet J 88:386-392. https://doi.org/10.1111/j.1751-0813.2010.00628.x

Del Carpio Munoz F, Syed FF, Noheria A et al (2011) Characteristics of premature ventricular complexes as correlates of reduced left ventricular systolic function: study of the burden, duration, coupling interval, morphology and site of origin of PVCs. J Cardiovasc Electrophysiol 22:791-798. https://doi.org/10.111 1/j.1540-8167.2011.02021.x

Duffee DF, Shen WK, Smith HC (1998) Suppression of frequent premature ventricular contractions and improvement of left ventricular function in patients with presumed idiopathic dilated cardiomyopathy. Mayo Clin Proc 73:430-433. https://doi.org/10.1016/ S0025-6196(11)63724-5

Haissaguerre M, Shoda M, Jais P (2002) Mapping and ablation of idiopathic ventricular fibrillation. Circulation 106:962-967. https:// doi.org/10.1161/01.CIR.0000027564.55739.B1

Hansen DE, Craig CS, Hondeghem LM (1990) Stretch-induced arrhythmias in the isolated canine ventricle. Evidence for the importance of mechanoelectrical feedback. Circulation 81:10941105. https://doi.org/10.1161/01.CIR.81.3.1094

Igarashi M, Tada H, Kurosaki K et al (2012) Electrocardiographic determinants of the polymorphic QRS morphology in idiopathic right ventricular outflow tract tachycardia. J Cardiovasc Electrophysiol 23:521-526. https://doi.org/10.111 1/j.1540-8167.2011.02232.x

Kanei Y, Friedman M, Ogawa N et al (2008) Frequent premature ventricular complexes originating from the right ventricular outflow tract are associated with left ventricular dysfunction. Ann Noninvasive Electrocardiol 13:81-85. https://doi.org/10.1111/j.1542474X.2007.00204.x

Kim YR, Nam GB, Kwon CH et al (2014) Second coupling interval of nonsustained ventricular tachycardia to distinguish malignant from benign outflow tract ventricular tachycardias. Hear Rhythm 11:2222-2230. https://doi.org/10.1016/j.hrthm.2014.08.012

Knecht S, Sacher F, Wright M et al (2011) Long-Term Follow-Up of Idiopathic Ventricular Fibrillation Ablation A Multicenter Study. J Am Coll Cardiol 54:522-528. https://doi.org/10.1016/j. jacc.2009.03.065

Kurosaki K, Nogami A, Shirai Y, Kowase S (2013) Positive QRS complex in lead $i$ as a malignant sign in right ventricular outflow tract tachycardia. Circ J 77:968-974. https://doi.org/10.1253/circj .CJ-12-0864
Lee YH, Zhong L, Roger VL et al (2014) Frequency, origin, and outcome of ventricular premature complexes in patients with or without heart diseases. Am J Cardiol 114:1373-1378. https://doi. org/10.1016/j.amjcard.2014.07.072

Mason JW, Koch FH, Billingham ME, Winkle RA (1978) Cardiac biopsy evidence for a cardiomyopathy associated with symptomatic mitral valve prolapse. Am J Cardiol 42:557-562. https:// doi.org/10.1016/0002-9149(78)90623-9

Oliveira MS, Muzzi R, Araújo LRB, et al (2012) Heart rate variability parameters of myxomatous mitral valve disease in dogs with and without heart failure obtained using 24-hour Holter electrocardiography. Vet Rec 170:622. https://doi.org/10.1136/vr.100202

Piccirillo G, Ogawa M, Song J et al (2009) Power spectral analysis of heart rate variability and autonomic nervous system activity measured directly in healthy dogs and dogs with tachycardia-induced heart failure. Heart Rhythm 6:546-552. https://doi.org/10.1016/j. hrthm.2009.01.006

Rhee KH, Jung JY, Rhee KS et al (2006) Tachycardiomyopathy induced by ventricular premature complexes: complete recovery after radiofrequency catheter ablation. Korean J Intern Med 21:213-217

Savage DD, Levy D, Garrison RJ et al (1983) Mitral valve prolapse in the general population. 3. Dysrhythmias: the framingham study. Am Heart J 106:582-586. https://doi.org/10.1016/00028703(83)90706-8

Sheldon SH, Gard JJ, Asirvatham SJ (2010) Premature ventricular contractions and non-sustained ventricular tachycardia: association with sudden cardiac death, risk stratification, and management strategies. Indian Pacing Electrophysiol J 10:357-371

Shepard JW, Garrison MW, Grither DA, Dolan GF (1985) Relationship of ventricular ectopy to oxyhemoglobin desaturation in patients with obstructive sleep apnea. Chest 88:335-340. https://doi. org/10.1378/chest.88.3.335

Shiraishi H, Ishibashi K, Urao N et al (2002) A case of cardiomyopathy induced by premature ventricular complexes. Circ J 66:10651067. https://doi.org/10.1253/circj.66.1065

Sun Y, Blom NA, Yu Y et al (2003) The influence of premature ventricular contractions on left ventricular function in asymptomatic children without structural heart disease: an echocardiographic evaluation. Int J Cardiovasc Imaging 19:295-299

Takemoto M, Yoshimura H, Ohba Y et al (2005) Radiofrequency catheter ablation of premature ventricular complexes from right ventricular outflow tract improves left ventricular dilation and clinical status in patients without structural heart disease. J Am Coll Cardiol 45:1259-1265. https://doi.org/10.1016/j.jacc.2004.12.073

Viskin S, Rosso R, Rogowski O, Belhassen B (2005) The "shortcoupled" variant of right ventricular outflow ventricular tachycardia: a not-so-benign form of benign ventricular tachycardia? J Cardiovasc Electrophysiol 16:912-916. https://doi.org/10.111 $1 /$ j.1540-8167.2005.50040.x 